\title{
Primeros registros de la comadreja de cola larga Mustela frenata (Carnivora: Mustelidae) para el departamento del Quindío, Andes Centrales de Colombia
}

\author{
First records of the long tail weasel Mustela frenata \\ (Carnivora: Mustelidae) for the department of Quindío, \\ Central Andes of Colombia
}

\author{
Rubén Darío Palacio ${ }^{1}$, Anderson Adrián Muñoz-Londoño², Hugo Mantilla-Meluk³ \\ Resumen
}

Se reportan los primeros registros acompañados de evidencia de la comadreja de cola larga Mustela frenata (Carnivora: Mustelidae) para el departamento del Quindío, en los Andes Centrales de Colombia. Los registros se refieren a ejemplares fotografiados en dos eventos independientes, que no fueron recolectados por falta de permisos oficiales. Los tres especímenes aquí reportados fueron avistados en jurisdicción del municipio de Filandia, en el «Distrito de Conservación de Suelos, Barbas-Bremen». Se discute sobre el estado de conocimiento y conservación de la comadreja de cola larga en el país y sobre la importancia del hallazgo en el contexto del conocimiento de la fauna local del Quindío. Finalmente localidades de colección de especímenes depositados en museos son utilizadas en la elaboración de un modelo predictivo de distribución de la especie en el que se evalúa la relevancia de estos nuevos reportes.

Palabras clave: Andes, Comadreja, Nuevos registros.

\begin{abstract}
We report the first records of the long tail weasel Mustela frenata (Carnivora: Mustelidae) accompanied by evidence, for the department of Quindío, in the Central Andes of Colombia. These records correspond to photographed animals in two independent events, not collected due to the lack of collecting permits, found at the municipio de Filandia; in the «Distrito de Conservación de Suelos, Barbas-Bremen». We discuss on the conservation status and knowledge of the long tail weasel in Colombia, as well as on the importance of these findings in the context of the knowledge of the local fauna in the department of Quindío. Finally, collecting localities of museum voucher specimens of $M$. frenata are used to construct a model of potential distribution for the species highlighting the importance of these new findings.
\end{abstract}

Keywords: Andes, New records, Weasel.

\section{Introducción}

La comadreja de cola larga Mustela frenata, es un carnívoro de tamaño mediano (180 a $220 \mathrm{~mm}$ ) y hábitos elusivos, que a pesar de su amplia distribución esta poco representado en colecciones científicas (Eisenberg 1989). A pesar de que se ha sugerido que la especie en Colombia tiene una amplia distribución (Izor y De La Torre 1978, Mesa-González 2006, Ramírez-Chávez y Mantilla-Meluk 2009), sus registros verificables corresponden a unas pocas localidades, estando la mayoría de sus reportes de presencia asociados con relatos anecdóticos, muchos de ellos referidos a ataques de depredación de aves de co-

Fundación Ecotonos y Departamento de Biología, Universidad Icesi, Cali, Colombia. e-mail: rdpalacio@ecotonos.org

Corporación Autónoma Regional del Quindío (CRQ), Armenia Quindío. e-mail: adrian2350@ hotmail.com

Programa de Biología, Universidad del Quindío, Armenia, Quindío. e-mail: hugo.mantillameluk@gmail.com

Fecha recepción: Noviembre 29, 2013 Fecha aprobación: Diciembre 22, 2013 Editor asociado: González JF 
rral. Pocos estudios se han conducido sobre este carnívoro en Colombia. En la literatura científica se cuenta con los aportes de Izor y De la Torre (1978) y Ramírez-Chaves y Mantilla-Meluk (2009), que incluyen análisis de la distribución de registros de la especie en Colombia y aspectos taxonómicos que separan a la comadreja de cola larga de su congenérico M. felipei en el país. En Colombia M. frenata ha sido registrada a la fecha para los departamentos de Antioquia, Boyacá, Caldas, Cauca, Cesar, Chocó, Cundinamarca, Huila, La Guajira, Magdalena, Nariño, Norte de Santander, Risaralda, Tolima y Valle del Cauca (Fawcett et al. 1996, Ramírez-Chávez et al. 2012, Solari et al. 2013). Ramírez-Chávez et al. (2012) analizaron 93 especímenes provenientes de más de 50 localidades a lo largo del país. A pesar de que las localidades altoandinas parecen ser de importancia para la especie [rango de distribución sugerido 1730 a 3050 m, Mesa-González (1997), aún hay departamentos en esta región, para los que la comadreja de cola larga no ha sido formalmente documentada, entre ellos el departamento del Quindío en los Andes Centrales de Colombia, donde a pesar de que la especie aparece reportada en varios informes técnicos de la Corporación Autónoma Regional del Quindío (CRQ), no se adiciona material que permita la verificación taxonómica de los mismos. De manera interesante, la especie no fue registrada en estudios recientes de mamíferos terrestres realizados en el norte del Quindío por Bedoya (2007), ni fue documentada en un monitoreo con cámaras trampa (Arroyave 2014 comm. pers.), en el que se estudió el uso de los corredores de conexión de Barbas-Bremen por la mastofauna de la parte norte del departamento. En este trabajo se reportan los primeros registros oficiales de la comadreja de cola larga $M$. frenata (Carnivora: Mustelidae) para el departamento del Quindío en los Andes Centrales de Colombia y se discute sobre la significancia de los mismos en el conocimiento de este carnívoro en el país.

\section{Métodos}

Modelo de distribución potencial en Maxent. Se recuperaron 49 de las coordenadas de las localidades colombianas presentadas para $M$. frenata por Ramírez-Chávez y Mantilla-Meluk (2009), a las que se les sumaron las localidades de presencia en este estudio con el fin de generar un modelo predictivo utilizando el algoritmo Maxent (Phillips et al. 2006). El modelo fue elaborado siguiendo los protocolos sugeridos por Phillips et al. (2006).

Rangos de elevación. Las localidades georreferenciadas fueron analizadas en sus rangos de distribución altitudinal utilizando como base un modelo de elevación digital (DEM) y la herramienta extract values to points en la aplicación Spatial Analyst de ArcGIS 10.1. Se reportó la media, desviación estándar, altura mínima y máxima de los registros de M. frenata utilizados.

\section{Resultados}

Primeros registros de Mustela frenata para el Quindío. Los registros corresponden a un ejemplar fotografiado (ejemplar 1, Figura 1), en el municipio de Filandia, Quindío, para el corredor Laureles (N 04 41' 36.3", W 75³7' 53.2", $1953 \mathrm{~m}$ ) que hace parte del sistema de corredores biológicos del Distrito de Conservación de Suelos, Barbas-Bremen. Este hallazgo se dio el día 14 de diciembre de 2012. Este ejemplar fue documentado durante un monitoreo de aves insectívoras de sotobosque, realizado por el autor principal, en compañía del ornitólogo local Pedro José Cardona. Al parecer, el individuo de $M$. frenata fue sacado de su madriguera y muerto por un perro doméstico que se encontraba en los corredores biológicos (Figura 2).

La localidad del segundo registro (ejemplar 2) corresponde a la Finca La Macenia, Vereda El Roble, Filandia, $1950 \mathrm{~m}$, área que también hace parte del Distrito de Conservación de Suelos BarbasBremen (Figuras 3 y 4). El encuentro con el espécimen tuvo lugar de manera ocasional por parte de uno de los autores (A Muñoz-Londoño) durante una actividad de campo correspondiente a un Monitoreo Fenológico de Flora Amenazada en el Departamento del Quindío y recolección de semillas. El lugar del encuentro corresponde al borde de un fragmento de bosque bien conservado (Figura 4), donde hay presencia de árboles de alto porte como el molinillo (Magnolia hernandezii), mediacaro (Pouteria lucuma), que se alterna con chusque (Chusquea sp), palmicho (Geonoma sp) y palma macana (Wettinia kalbreyeri). Se observaron dos individuos adultos, que se asume conforman una pareja en cercanías de 


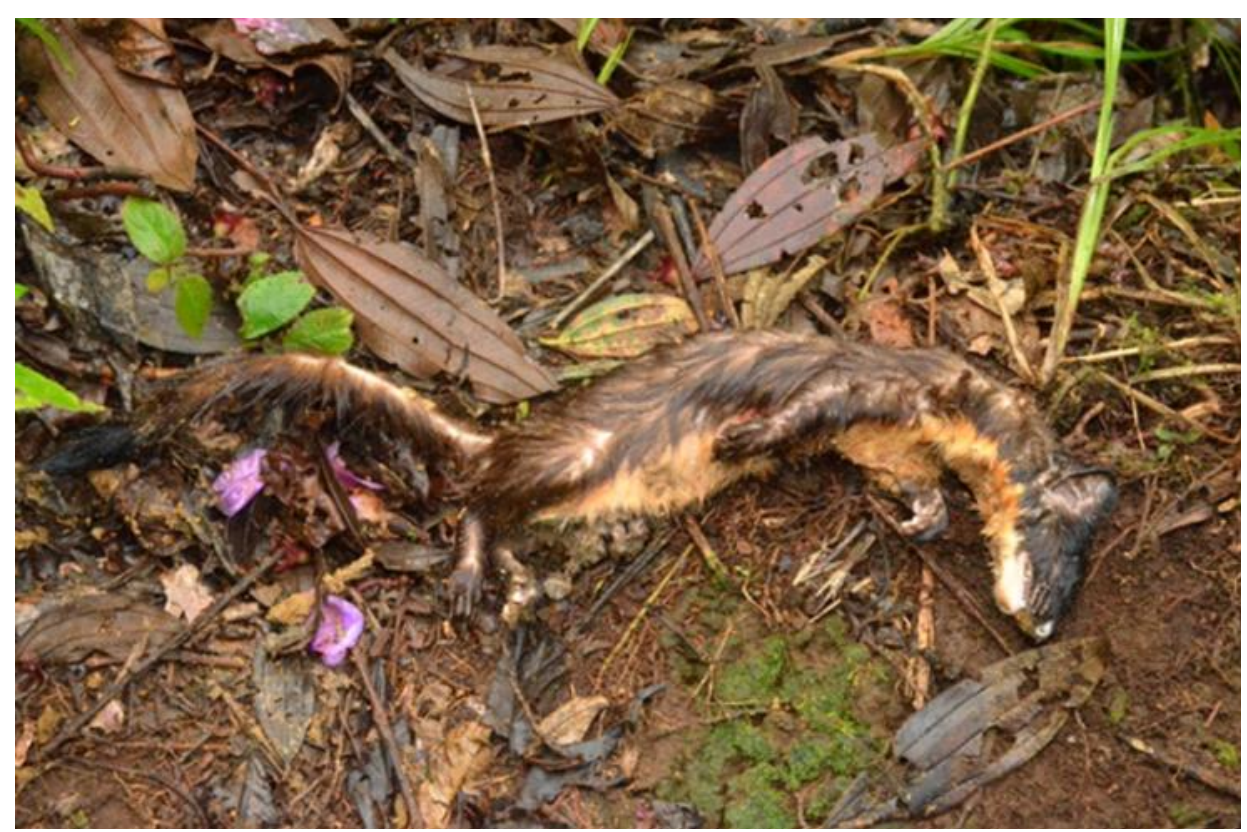

Figura 1. Ejemplar 1 de Mustela frenata registrado en los corredores de Barbas-Bremen, Filandia, Quindío, muerto por un perro fuera de su madriguera (fotografía RD Palacio).

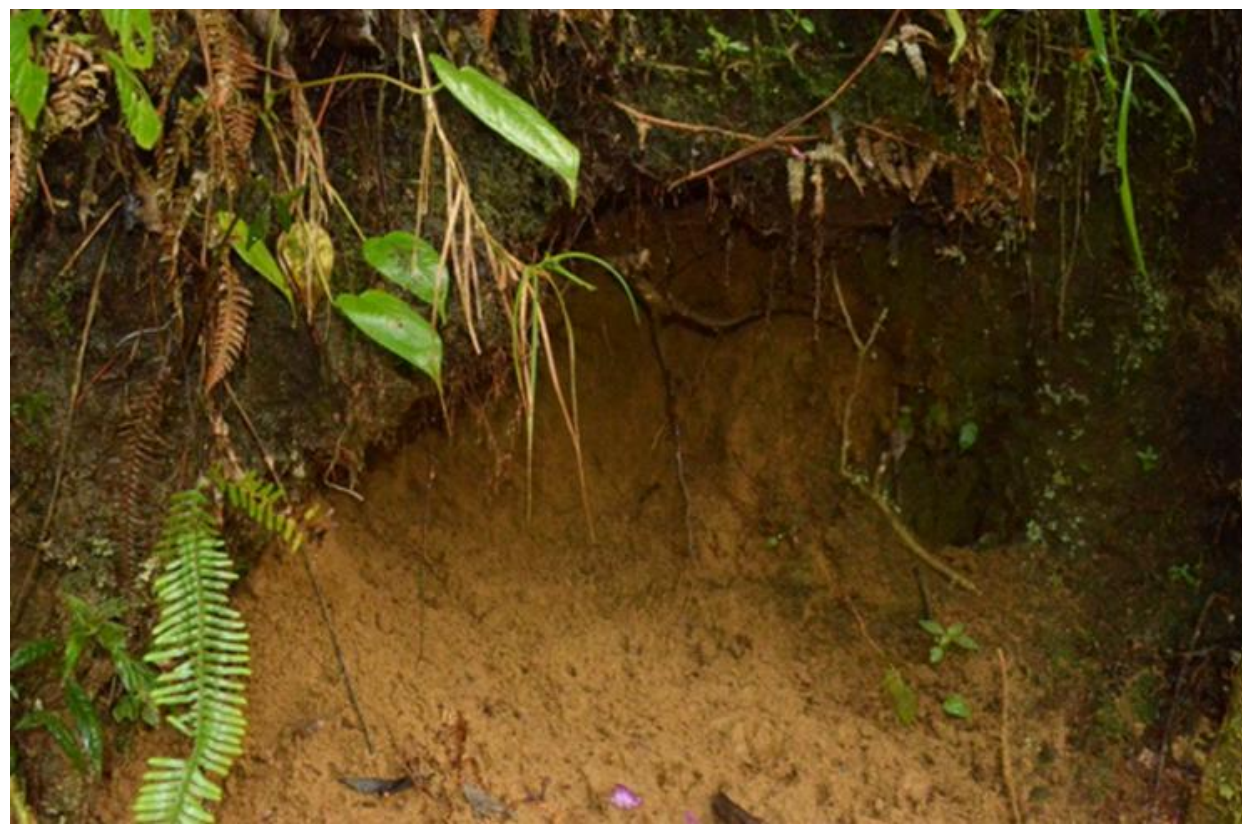

Figura 2. Entrada de la madriguera del ejemplar 1 de $M$. frenata fotografiado en los corredores de Barbas-Bremen.

una madriguera ubicada en raíz de un árbol con buen fuste y abundante hojarasca, líquenes y briófitos lo que la hace casi imperceptible. Este encuentro se dio el 11 de septiembre de 2014, a las 02:20 pm.

Infortunadamente los ejemplares no pudieron ser recolectados para ser depositados en una colección científica debido a la ausencia de permisos de recolección de mamíferos, acatando el Decreto 1376 de
2013 del Ministerio de Ambiente y Desarrollo Sostenible (Por el cual se reglamenta el permiso de recolección de especímenes de especies silvestres de la diversidad biológica con fines de investigación científica no comercial), no fue posible capturar los individuos, hasta tanto la investigación cuente con la normatividad vigente.

Determinación taxonómica. Para Colombia, la 


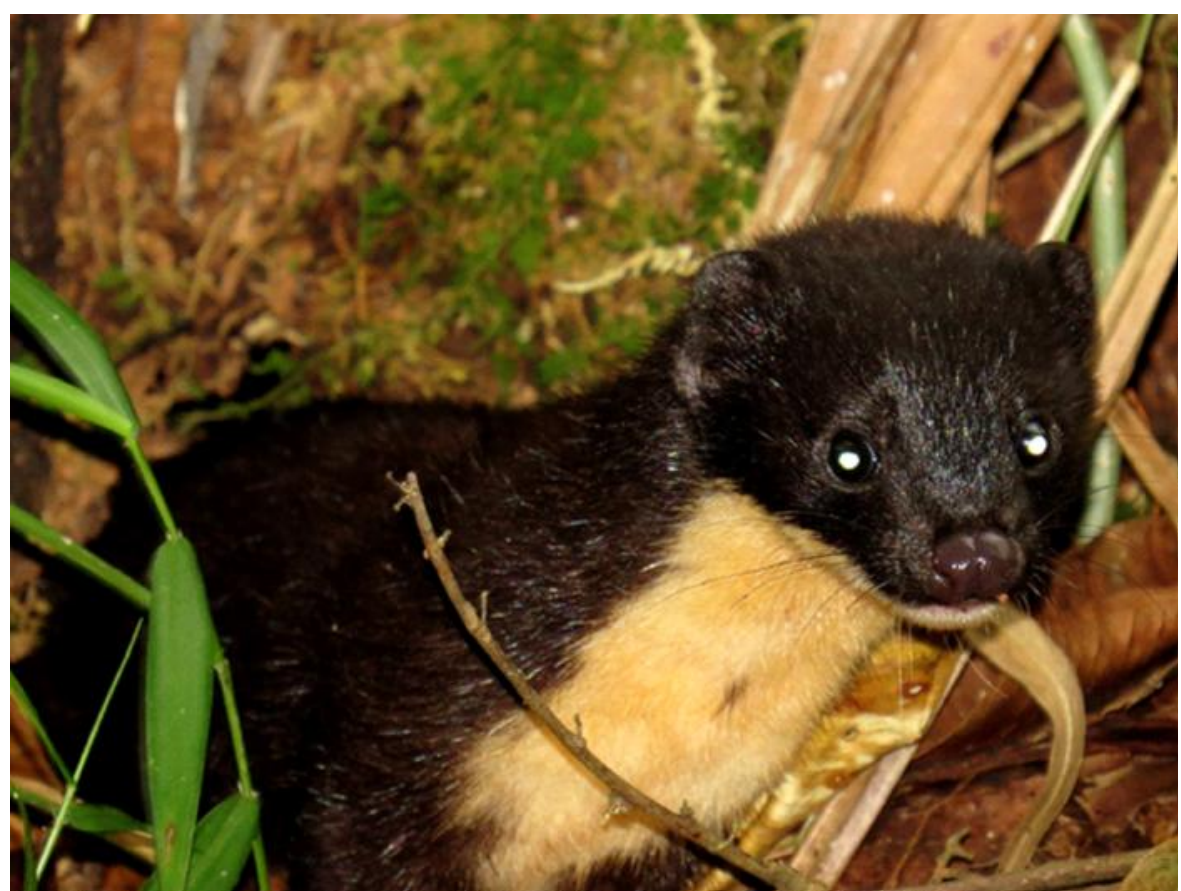

Figura 3. Ejemplar 2 de M. frenata fotografiado en la Finca La Macenia, Vereda El Roble, Filandia, Quindío. Se puede apreciar la ausencia de mancha gular conspicua y manchas faciales típicas de la congenérica M. felipei (fotografía A Muñoz-Londoño).

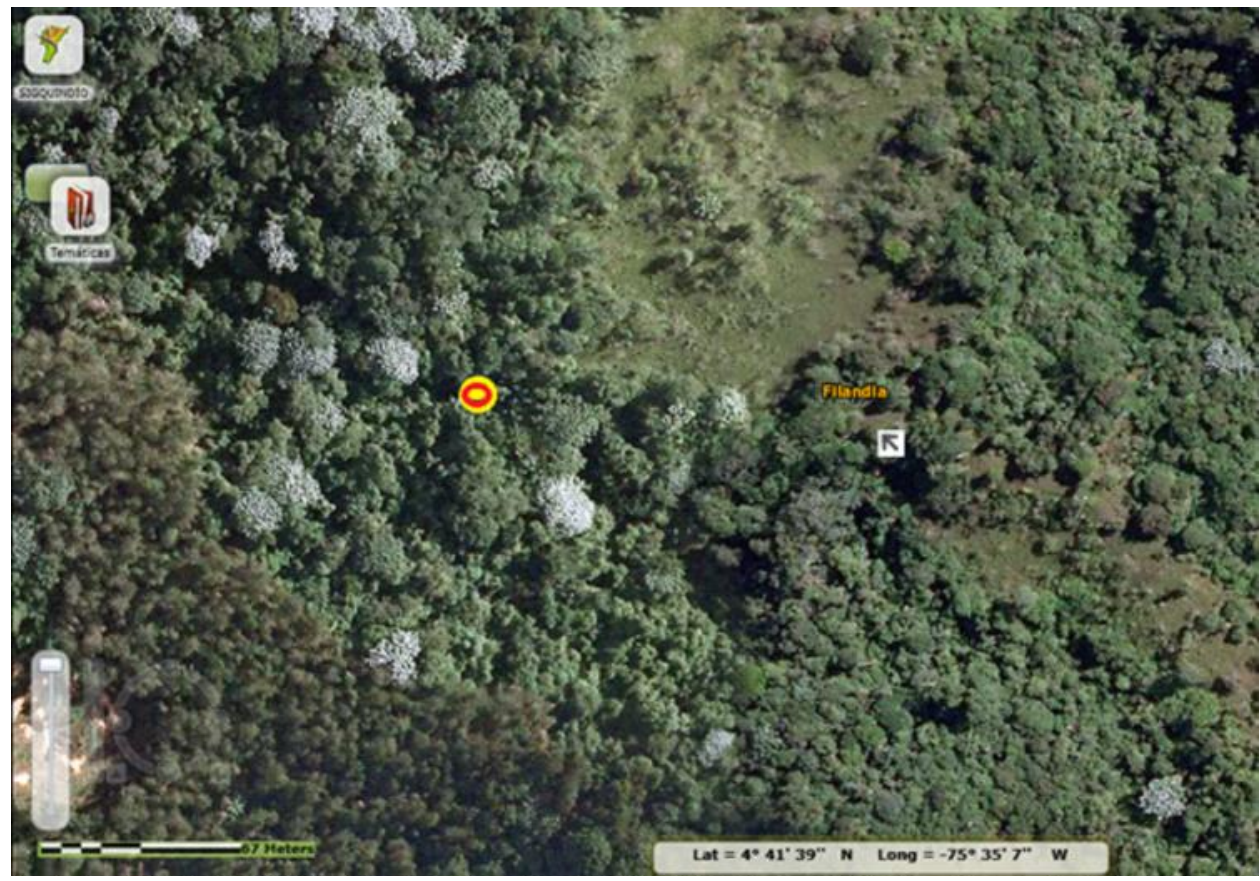

Figura 4. Fotografía aérea en la que se señala el lugar de la observación del ejemplar 2 de $M$. frenata proveniente de Filandia, Quindío (símbolo amarillo) y las características de la cobertura boscosa.

distribución de M. frenata se sobrepone en los Andes Centrales con la distribución de la comadreja colombiana M. felipei, especie más cercana en morfología externa (Ramírez-Chávez y Mantilla-Meluk
2009). Sin embargo, estas dos especies son fácilmente distinguibles por caracteres externos entre los que se cuentan para $M$. frenata: a) Planta de las patas cubiertas con pelos; b) Cola larga con el borde distal 
negro; c) Manchas faciales presentes (excepto en ejemplares muy jóvenes); d) Mancha gular ausente (Ramírez-Chávez y Mantilla-Meluk 2009) (Figuras 1 y 3), todos estos caracteres fueron documentados para los tres ejemplares de Filandia, Quindío.

Ecología de la especie. Es poco lo que se conoce sobre la ecología de la comadreja de cola larga en el país. A nuestro saber, los estudios de ecología de la especie se refieren a dos trabajos de grado separados entre sí por casi 20 años. Mesa-González (1997) basada en telemetría, estudió los patrones de uso del hábitat de un individuo rastreado en la región del Tambo, Cauca, encontrando que el área utilizada por el mismo estuvo entre 12 y 16 hectáreas con fluctuaciones en uso asociadas con la disponibilidad de recursos alimenticios, que básicamente correspondieron a pequeños mamíferos domésticos y semi-domésticos (pollos, cuyes y gatos). Concluye esta autora que $M$. frenata es un animal de movimientos diurnos, que prefiere áreas de cobertura boscosa donde es menos vulnerable a predadores naturales y al hombre (Mesa-González 1997). Por su parte Hiller (2004), estudio el tamaño poblacional de $M$. frenata en el humedal La Conejera, en el área de Bogotá, en la Cordillera Oriental, concluyendo que el tamaño poblacional de este carnívoro para esa localidad es bajo y que del área cubierta por el humedal, la comadreja de cola larga prefiere los sectores más preservados con cobertura de árboles.

Estado de conservación y situación en el Quindío. La comadreja de cola larga está clasificada como especie de preocupación menor en términos de conservación por la Unión Internacional para la Conservación de la Naturaleza (Reid y Helgen 2008). Este carnívoro es usualmente común en los últimos estados serales o en ecotonos donde la diversidad de presas es aparentemente mayor (Wilson y Ruff 1999). Estudios de telemetría apuntan a que las comadrejas de cola larga son sensibles a la trasformación de los ecosistemas derivada de actividades agrícolas que resultan en la fragmentación de las coberturas naturales afectando la conectividad de las especies (Gehring y Swihart 2004). Es por esta razón que el hallazgo de $M$. frenata en los corredores de conectividad de Barbas-Bremen se podría asociar a la recuperación de las condiciones naturales en un área que como esta, hasta hace una década constituyó terrenos de potreros. El área de Barbas-
Bremen fue declarada Parque Regional Natural Barbas-Bremen, en el año 2007, cambiando su estatus a Distrito de Conservación de Suelos según el acuerdo N 17 de 2011 de la Corporación Autónoma Regional de Risaralda (CARDER). Su extensión cubre un área de 9.651 hectáreas, de las cuales 5.335 se hayan en territorio del Quindío y 4.316 ha en el departamento de Risaralda. El área del parque incluye un gradiente de elevación entre los 1.650 y 2.600 msnm con una temperatura media anual que oscila entre $\operatorname{los} 12^{\circ} \mathrm{C}$ y $18^{\circ} \mathrm{C}$. Adicionalmente, el área de reserva incluye la masa boscosa más importante en biodiversidad para el Quindío y hace parte de la zona amortiguadora del Parque de Los Nevados, con un Área de Importancia para la Conservación de Aves (AICA) en el área de conservación Bremen La Popa.

Registros de la especie y modelamiento de nicho. Nuestro modelo basado en 49 localidades de registro de la especie, señala que la comadreja de cola larga en Colombia es una especie típicamente altoandina y que su presencia se extiende a los sistemas montañosos independientes a los Andes en Colombia, en la Sierra Nevada de Santa Marta (con un registro proveniente de San Lorenzo) y la Serranía de la Macarena ( $\sin$ registros conocidos de $M$. frenata). La mayoría de los registros provienen de la Cordillera Central $(\mathrm{N}=29)$, seguidos por registros en la Cordillera Oriental ( $\mathrm{N}=19)$. Todos los registros de localidades fueron en altitudes mayores a los $863 \mathrm{~m}$, con un valor medio de $2086 \mathrm{~m}$ (DE= $574 \mathrm{~m}$ ). El valor máximo de elevación registrado según nuestros análisis es de $2924 \mathrm{~m}$. El modelo presenta un valor de omisión nulo (Figura 5).

\section{Conclusiones}

Concluimos que es importante intensificar los estudios mastozoológicos en el departamento del Quindío con el fin de tener una mayor resolución sobre la composición de la mastofauna local y que estos estudios en la medida de las posibilidades estén sustentados por evidencia que permita la verificación de las hipótesis taxonómicas y de distribución propuestas. Se deriva de nuestra investigación que $M$. frenata, a pesar de ser una especie reportada como común y de amplia distribución en el país, es un carnívoro poco conocido en el contexto nacional cuyas poblaciones pueden estar sufriendo presiones de se- 


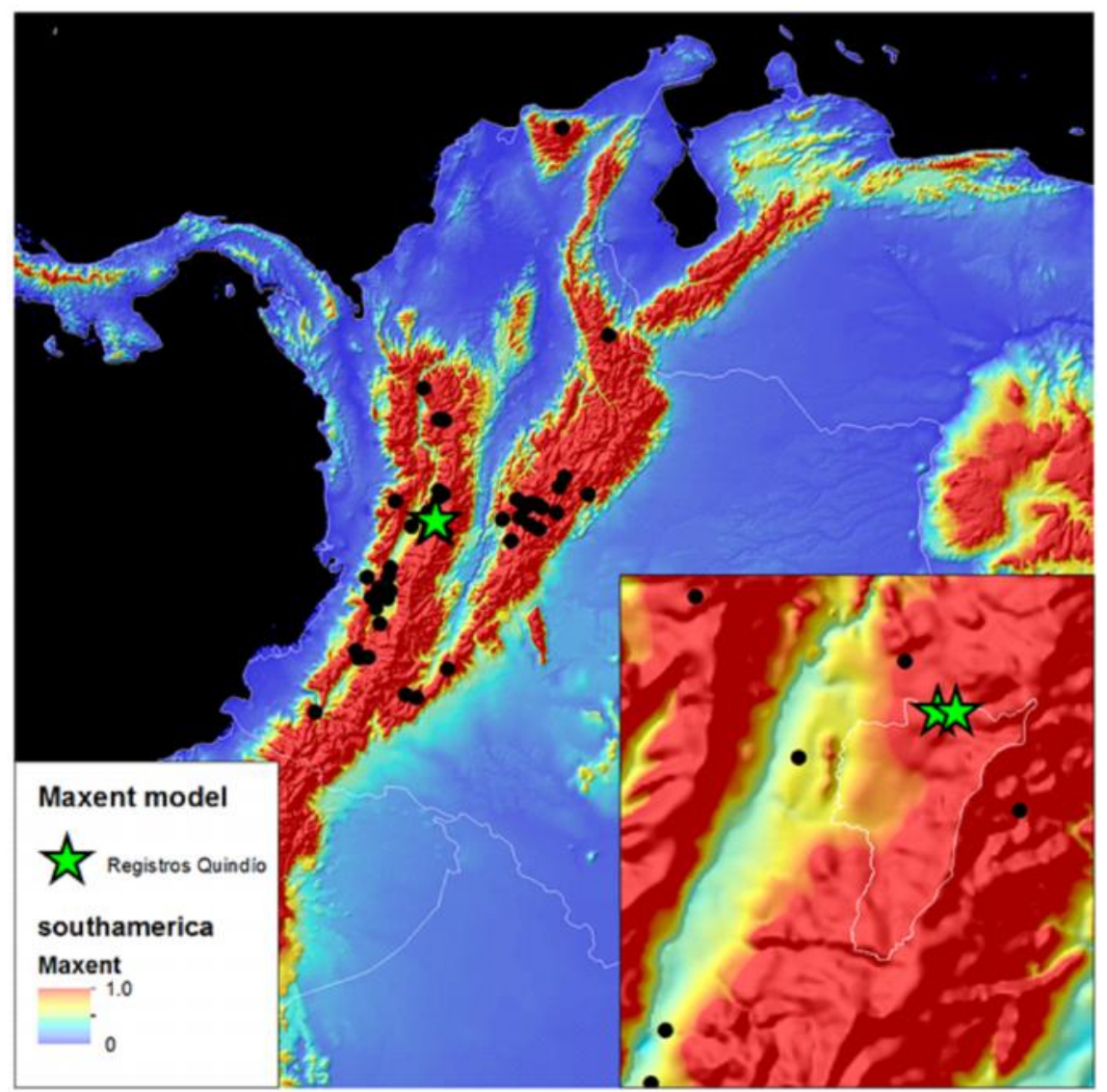

Figura 5. Modelo de distribución potencial producido para M. frenata en Colombia, basado en 49 localidades de registro de presencia de la especie derivados de Ramírez-Chávez y Mantilla-Meluk (2009) (puntos negros), más registros adicionados en este trabajo de Filandia, Quindío (estrellas verdes). En el recuadro detalle del área del departamento del Quindío.

lección fuertes, e incluso la extinción local al ser organismos cuyos registros, como en este caso, provienen de áreas relativamente bien conservadas. Comunicación personal con los pobladores del área refieren una disminución en los avistamientos de este carnívoro. Se comenta que la disminución de fincas pequeñas que han dado paso a latifundios con monocultivos de plátano y café hacen que los encuentros con la comadreja sean menos frecuentes, porque en el pasado las comadrejas visitaban las fincas pequeñas que usualmente tenían aves de corral desplazándose libremente en su extensión. Hoy en día la cría de aves de corral se da de manera tecnificada, con galpones que aglutinan un número significativo de aves. Uno de los autores (H Mantilla-Meluk) registró un encuentro casual con una comadreja de cola larga en inmediaciones de un galpón de cría de aves en el municipio de Quimbaya, en la vereda el Laurel, camino a la reserva «La Montaña del Ocaso», admi- nistrada por la Universidad del Quindío, área que aún posee coberturas boscosas relativamente bien conservadas.

Finalmente, se valida la importancia de iniciativas como los corredores biológicos del BarbasBremen que han resultado no solo en la recuperación de cobertura boscosa, sino también las relaciones ecosistémicas, que como en este caso, han permitido el establecimiento de una especie ubicada en el tope de la cadena alimenticia. Se recomienda a las autoridades ambientales locales el desarrollo de acciones tendientes a preservar el tejido biológico recuperado en los corredores del Barbas-Bremen.

\section{Literatura citada}

Bedoya JJ. 2007. Valoración inicial del potencial de conectividad de corredores biológicos por medio de mamíferos terrestres medianos y grandes en un bosque subandino. 
Tesis. Bogotá: Universidad Javeriana.

Eisenberg JF. 1989. Mammals of the Neotropics. Vol 1. Chicago: University of Chicago Press. Pp. 274-5.

Fawcett D, Rojas-Dias V, Montero H. 1996. Colombian Weasel. Small Carniv Conservat. 14: 7-10.

Gehring TM, Swiharat YRK. 2004. Home range and movements of long-tailed weasels in a landscape fragmented by agriculture. J Mammal. 85: 79-86.

Hiller H. 2004. Tamaño poblacional y distribución de la comadreja de cola larga (Mustela frenata) en el humedal de la conejera, Bogotá, Colombia. Tesis de grado. Bogotá: Departamento de Biología, Pontificia Universidad Javeriana; 85 pp.

Izor RJ, De La Torre L. 1978. A new species of weasel (Mustela) from the highlands of Colombia, with comments on the evolution and distribution of South America weasels. $J$ Mammal. 59: 92-102.

Mesa-González E. 1997. Rango de acción, uso de hábitat y anotaciones sobre conocimiento popular del chucuro (Mustela frenata), en una zona del Tambo, Cauca. Tesis de grado para optar al título de biólogo. Bogotá: Facultad de Ciencias, Departamento de Biología, Universidad Nacional de Colombia. 63 pp.
Mesa-González E. 2006. Comadreja colombiana Mustela felipei. En: Rodríguez-Mahecha JV, Alberico M, Trujillo F, Jorgenson J (eds.). Libro Rojo de los Mamíferos de Colombia. Bogotá: Serie Libros Rojos de Especies Amenazadas de Colombia. Conservación Internacional Colombia, Ministerio de Ambiente, Vivienda y Desarrollo Territorial. pp: 139-45, 272.

Phillips SJ, Anderson R, Shapire RE. 2006. Maximum entropy modeling of species geographic distributions. Ecol Model. 190: 231-59.

Ramírez-Chávez HE, Mantilla-Meluk H. 2009. Nuevo registro de la comadreja colombiana Mustela felipei (Carnivora: Mustelidae), con notas sobre su distribución y conservación. Mastozool Neotrop. 16 (2): 379-88.

Ramírez-Cháves HE, Mulder KP, Marin D, Pérez WA, MartínezÁrias VM. 2012. Has Colombian Weasel Mustela felipei been overlooked in collections? Small Carniv Conservat. 47: 30-4.

Reid F, Helgen K. 2008. Mustela frenata. The IUCN Red List of Threatened Species. Version 2014.2. Descargado el 31 agosto de 2014. www.iucnredlist.org

Wilson DE, Ruff SY. 1999. The Smithsonian Book of North American Mammals. Vancouver: University of British Columbia Press; 750 pp. 